\title{
Passivity Analysis of Complex Delayed Dynamical Networks with Output Coupling
}

\author{
Yan-Fang Kang, ${ }^{1}$ Lu-Xian Fang, ${ }^{2}$ Yue-Hui Zhao, ${ }^{2}$ and Shun-Yan Ren ${ }^{3}$ \\ ${ }^{1}$ School of Economics, Wuhan University of Technology, Wuhan 430070, China \\ ${ }^{2}$ The College of Post and Telecommunication, Wuhan Institute of Technology, Wuhan 430070, China \\ ${ }^{3}$ School of Computer Science and Software Engineering, Tianjin Polytechnic University, Tianjin 300387, China \\ Correspondence should be addressed to Yue-Hui Zhao; zhyh83@tom.com
}

Received 12 May 2014; Revised 29 August 2014; Accepted 30 August 2014

Academic Editor: Zhichun Yang

Copyright (C) 2015 Yan-Fang Kang et al. This is an open access article distributed under the Creative Commons Attribution License, which permits unrestricted use, distribution, and reproduction in any medium, provided the original work is properly cited.

\begin{abstract}
A new complex dynamical network model with output coupling is proposed. This paper is concerned with input passivity and output passivity of the proposed network model. By constructing new Lyapunov functionals, some sufficient conditions ensuring the input passivity and output passivity are obtained. Finally, two numerical examples are provided to demonstrate the effectiveness of the proposed results.
\end{abstract}

\section{Introduction}

Recently, there has been increasing interest in the study of complex dynamical networks. The main reason is that many practical systems can be characterized by various models of complex networks. It is well known that one of the most significant and interesting dynamical phenomena of complex networks is the synchronization of systems. Many interesting results on synchronization have been derived for various complex networks [1-10]. But, it should be noticed that the complex networks with state coupling were considered in these papers.

To our knowledge, Jiang et al. [11] first introduced a complex network model with output coupling without time delays. Some conditions for synchronization were established based on the Lyapunov stability theory. However, time delays always exist in complex networks due to the finite speeds of transmission and/or the traffic congestion, and most of delays are notable. So it is crucial for us to take the delay into the consideration when we study complex networks. Practically, many phenomena in nature can be modeled as complex networks with output coupling. The cooperative control problem of multiple agents has received much attention recently since it has challenging features and many applications, for example, large object moving, formation control, rescue mission, and satellite clustering. It is well known that the state of agent is difficult to be observed or measured because of technology limitations and environmental disturbances. For instance, the measuring of velocity is more difficult than that of position. In some circumstances, the information of velocity is unavailable for agents [12]. Therefore, it is quite necessary to design protocols based on the output variables. In this case, the closed-loop systems can be described by the complex networks with output coupling. Hence, study of complex networks with output coupling is very interesting and important in both theory and application. A complex delayed dynamical network model with output coupling was proposed in $[13,14]$. Wang and $\mathrm{Wu}[13]$ investigated the output synchronization of the proposed network model, and some criteria on local and global exponential output synchronization were derived.

Passivity [15-33] is an important concept of system theory and provides a nice tool for analyzing the stability of systems and has found applications in diverse areas such as stability, complexity, signal processing, chaos control and synchronization, and fuzzy control. Many researchers have studied the passivity of fuzzy systems [19-22] and neural networks [23-28]. Liang et al. [20] discussed the passivity and passification problems for a class of uncertain stochastic fuzzy systems with time-varying delays. In [26] Song et al. 
investigated the passivity for a class of discrete-time stochastic neural networks with time-varying delays, and a delaydependent passivity condition was obtained by constructing proper Lyapunov-Krasovskii functional. However, there are few work on the passivity of complex networks [29, 30, 32, 33]. In $[29,30]$, Yao et al. obtained some sufficient conditions on passivity properties for linear (or linearized) complex networks with and without coupling delays (constant delay). However, in practical evolutionary processes of the networks, absolute constant delay may be scarce and delays are frequently varied with time. Therefore, it is important to further study the passivity of complex networks with timevarying delays. Wang et al. [32] considered input passivity and output passivity for a generalized complex network with nonlinear, time-varying, nonsymmetric, and delayed coupling. By constructing some suitable Lyapunov functionals, several sufficient conditions ensuring input passivity and output passivity were derived. To the best of our knowledge, the input passivity and output passivity of complex delayed dynamical network model with output coupling have not yet been established. Therefore, it is interesting to study the input passivity and output passivity of complex delayed dynamical network model with output coupling.

Motivated by the above discussions, we propose a new complex delayed dynamical network model with output coupling. The objective of this paper is to study the input and output passivity of the proposed network model. Some sufficient conditions ensuring input passivity and output passivity are obtained by Lyapunov functional method.

The rest of this paper is organized as follows. A new complex network model is introduced and some useful preliminaries are given in Section 2. Several input and output passivity criteria are established in Section 3. In Section 4, two numerical examples are given to illustrate the effectiveness of the proposed results. Conclusions are finally given in Section 5 .

\section{Network Model and Preliminaries}

Let $R^{n}$ be the $n$-dimensional Euclidean space, and let $R^{n \times m}$ be the space of $n \times m$ real matrices. $P \geqslant 0(P \leqslant 0)$ means that matrix $P$ is real symmetric and semipositive (seminegative) definite. $P>0(P<0)$ means that matrix $P$ is real symmetric and positive (negative) definite. $I_{n}$ denotes the $n \times n$ identity matrix. $B^{T}$ denotes the transpose of a square matrix B. $C\left([-\tau, 0], R^{n}\right)$ is a Banach space of continuous functions mapping the interval $[-\tau, 0]$ into $R^{n}$ with the norm $\|\phi\|_{\tau}=$ $\sup _{-\tau \leqslant \theta \leqslant 0}\|\phi(\theta)\|$, where $\|\cdot\|$ is the Euclidean norm.

In this paper, we consider a complex delayed dynamical network consisting of $N$ identical nodes with diffusive and output coupling. The mathematical model of the coupled network can be described as follows:

$$
\begin{gathered}
\dot{x}_{i}(t)=f\left(x_{i}(t)\right)+\frac{a}{k_{i}^{\beta_{\omega}}} \sum_{j=1}^{N} L_{i j} \Gamma y_{j}(t-\tau(t))+B_{i} u_{i}(t), \\
y_{i}(t)=C_{i} x_{i}(t)+D_{i} u_{i}(t)
\end{gathered}
$$

where $i=1,2, \ldots, N . \tau(t)$ is the time-varying delay with $0 \leqslant$ $\tau(t) \leqslant \tau$.

The function $f(\cdot)$, describing the local dynamics of the nodes, is continuously differentiable and capable of producing various rich dynamical behaviors, $x_{i}(t)=\left(x_{i 1}(t), x_{i 2}(t)\right.$, $\left.\ldots, x_{i n}(t)\right)^{T} \in R^{n}$ is the state variable of node $i, y_{i}(t) \in R^{n}$ is the output of node $i, u_{i}(t) \in R^{n}$ is the input vector of node $i, B_{i}$, $C_{i}$, and $D_{i}$ are known matrices with appropriate dimensions, $\Gamma \in R^{n \times n}$ is inner-coupling matrix, which describes the individual coupling between two connected nodes of the network, $a>0$ represents the overall coupling strength, $k_{i}$ is the degree of node $i, \beta_{\omega}$ is a tunable weight parameter, and the real matrix $L=\left(L_{i j}\right)_{N \times N}$ is a symmetric matrix with diagonal entries $L_{i i}=-k_{i}$ and off-diagonal entries $L_{i j}=1$ if node $i$ and node $j$ are connected by a link, and $L_{i j}=0$ otherwise.

In this paper, we always assume that complex network (1) is connected. Let $x(t)=\left(x_{1}^{T}(t), x_{2}^{T}(t), \ldots, x_{N}^{T}(t)\right)^{T}, y(t)=$ $\left(y_{1}^{T}(t), y_{2}^{T}(t), \ldots, y_{N}^{T}(t)\right)^{T}$, and $u(t)=\left(u_{1}^{T}(t), u_{2}^{T}(t), \cdots\right.$, $\left.u_{N}^{T}(t)\right)^{T}$. The initial condition associated with the complex network (1) is given in the form

$$
\begin{gathered}
x(0)=x_{0}, \quad x_{0}=\left(x_{10}^{T}, x_{20}^{T}, \ldots, x_{N 0}^{T}\right)^{T}, \quad x_{i 0} \in R^{n}, \\
y(s)=\Phi(s), \quad s \in[-\tau, 0], \quad \phi_{i} \in C\left([-\tau, 0], R^{n}\right), \\
\Phi(s)=\left(\phi_{1}^{T}(s), \phi_{2}^{T}(s), \ldots, \phi_{N}^{T}(s)\right)^{T}, \quad i=1,2, \ldots, N .
\end{gathered}
$$

Next, we give several useful definitions.

Definition 1 (see [32]). Complex network (1) is called input passive if there exist two constants $\gamma>0$ and $\beta \in R$ such that

$$
2 \int_{0}^{t_{p}} y^{T}(s) u(s) d s \geqslant-\beta^{2}+\gamma \int_{0}^{t_{p}} u^{T}(s) u(s) d s
$$

for all $t_{p} \geqslant 0$.

Definition 2 (see [32]). Complex network (1) is called output passive if there exist two constants $\gamma>0$ and $\beta \in R$ such that

$$
2 \int_{0}^{t_{p}} y^{T}(s) u(s) d s \geqslant-\beta^{2}+\gamma \int_{0}^{t_{p}} y^{T}(s) y(s) d s
$$

for all $t_{p} \geqslant 0$.

Definition 3 (see [34]). Let $A=\left(a_{i j}\right)_{m \times n} \in R^{m \times n}$, and let $B=\left(b_{i j}\right)_{p \times q} \in R^{p \times q}$. Then the Kronecker product (or tensor product) of $A$ and $B$ is defined as the matrix

$$
A \otimes B=\left[\begin{array}{cccc}
a_{11} B & a_{12} B & \cdots & a_{1 n} B \\
a_{21} B & a_{22} B & \cdots & a_{2 n} B \\
\vdots & \vdots & \cdots & \vdots \\
a_{m 1} B & a_{m 2} B & \cdots & a_{m n} B
\end{array}\right] \in R^{m p \times n q}
$$


The Kronecker product has the following properties:

(1) $(A \otimes B)^{T}=A^{T} \otimes B^{T}$;

(2) $(\alpha A) \otimes B=A \otimes(\alpha B)$;

(3) $(A+B) \otimes C=A \otimes C+B \otimes C ;$

(4) $(A \otimes B)(C \otimes D)=(A C) \otimes(B D)$,

where $\alpha \in R, C$, and $D$ are matrices with suitable dimensions.

\section{Main Results}

In this section, we shall investigate the input passivity and output passivity of the complex delayed dynamical networks with output coupling.

In $[32,35]$, authors make the assumption that the function $f(\cdot)$ is in the QUAD function class. In this paper, we make similar assumptions.

(A1) There exist a positive definite diagonal matrix $P=\operatorname{diag}\left(p_{1}, p_{2}, \ldots, p_{n}\right)$ and a diagonal matrix $\Delta=$ $\operatorname{diag}\left(\delta_{1}, \delta_{2}, \ldots, \delta_{n}\right)$ such that $f$ satisfies the following inequality:

$$
x^{T} P(f(x)-\Delta x) \leqslant-\eta x^{T} x,
$$

for some $\eta>0$ and all $x \in R^{n}$.

For the convenience, we denote

$$
\begin{gathered}
\widehat{P}=\operatorname{diag}(P, P, \ldots, P), \quad \widehat{\Delta}=\operatorname{diag}(\Delta, \Delta, \ldots, \Delta), \\
B=\operatorname{diag}\left(B_{1}, B_{2}, \ldots, B_{N}\right), \quad C=\operatorname{diag}\left(C_{1}, C_{2}, \ldots, C_{N}\right), \\
D=\operatorname{diag}\left(D_{1}, D_{2}, \ldots, D_{N}\right) .
\end{gathered}
$$

In the following, we first give two input passivity criteria.

Theorem 4. Let (A1) hold, and let $\dot{\tau}(t) \leqslant \sigma<1$. The complex network (1) is input passive if there exist matrix $Q \geqslant 0$ and a scalar $\gamma>0$ such that

$$
\left(\begin{array}{ccc}
W_{1} & a \widehat{P}(G \otimes \Gamma) & W_{2} \\
a(G \otimes \Gamma)^{T} \widehat{P} & -(1-\sigma) Q & 0 \\
W_{2}^{T} & 0 & W_{3}
\end{array}\right) \leqslant 0
$$

where

$$
\begin{gathered}
W_{1}=-2 \eta I_{n N}+2 \widehat{P} \widehat{\Delta}+C^{T} Q C, \\
W_{2}=\widehat{P} B+C^{T} Q D-C^{T}, \\
W_{3}=-\left(D+D^{T}-\gamma I_{n N}-D^{T} Q D\right) .
\end{gathered}
$$

Proof. For convenient analysis, we let

$$
G_{i j}=\frac{L_{i j}}{k_{i}^{\beta_{\omega}}} .
$$

Then, complex network (1) can be rewritten as follows:

$$
\begin{gathered}
\dot{x}_{i}(t)=f\left(x_{i}(t)\right)+a \sum_{j=1}^{N} G_{i j} \Gamma y_{j}(t-\tau(t))+B_{i} u_{i}(t), \\
y_{i}(t)=C_{i} x_{i}(t)+D_{i} u_{i}(t),
\end{gathered}
$$

where $i=1,2, \ldots, N . G=\left(G_{i j}\right)_{N \times N}$ is a coupling matrix, accounting for the topology of complex dynamical network. We can rewrite system (12) in a compact form as follows:

$$
\begin{gathered}
\dot{x}(t)=F(x(t))+a(G \otimes \Gamma) y(t-\tau(t))+B u(t), \\
y(t)=C x(t)+D u(t),
\end{gathered}
$$

where

$$
\begin{gathered}
x(t)=\left(x_{1}^{T}(t), x_{2}^{T}(t), \ldots, x_{N}^{T}(t)\right)^{T}, \\
u(t)=\left(u_{1}^{T}(t), u_{2}^{T}(t), \ldots, u_{N}^{T}(t)\right)^{T}, \\
y(t)=\left(y_{1}^{T}(t), y_{2}^{T}(t), \ldots, y_{N}^{T}(t)\right)^{T}, \\
F(x(t))=\left(f^{T}\left(x_{1}(t)\right), f^{T}\left(x_{2}(t)\right), \ldots, f^{T}\left(x_{N}(t)\right)\right)^{T}, \\
y(t-\tau(t)) \\
=\left(y_{1}^{T}(t-\tau(t)), y_{2}^{T}(t-\tau(t)), \ldots, y_{N}^{T}(t-\tau(t))\right)^{T} .
\end{gathered}
$$

Construct Lyapunov functional for system (13) as follows:

$$
V(t)=x^{T}(t) \widehat{P} x(t)+\int_{t-\tau(t)}^{t} y^{T}(\alpha) Q y(\alpha) d \alpha .
$$

The derivative of $V(t)$ satisfies

$$
\begin{aligned}
\dot{V}(t)= & 2 x^{T}(t) \widehat{P} \dot{x}(t)+y^{T}(t) Q y(t) \\
& -(1-\dot{\tau}(t)) y^{T}(t-\tau(t)) Q y(t-\tau(t)) \\
\leqslant & 2 x^{T}(t) \widehat{P} \dot{x}(t)+y^{T}(t) Q y(t) \\
& -(1-\sigma) y^{T}(t-\tau(t)) Q y(t-\tau(t)) \\
= & 2 x^{T}(t) \widehat{P} F(x(t))+2 a x^{T}(t) \widehat{P}(G \otimes \Gamma) y(t-\tau(t)) \\
& +2 x^{T}(t) \widehat{P} B u(t)+y^{T}(t) Q y(t) \\
& -(1-\sigma) y^{T}(t-\tau(t)) Q y(t-\tau(t)) .
\end{aligned}
$$

Then, we can get

$$
\begin{aligned}
& \dot{V}(t)-2 y^{T}(t) u(t)+\gamma u^{T}(t) u(t) \\
& \quad \leqslant 2 x^{T}(t) \widehat{P} F(x(t))+2 a x^{T}(t) \widehat{P}(G \otimes \Gamma) y(t-\tau(t))
\end{aligned}
$$




$$
\begin{aligned}
& +2 x^{T}(t) \widehat{P} B u(t)+y^{T}(t) Q y(t) \\
& -(1-\sigma) y^{T}(t-\tau(t)) Q y(t-\tau(t)) \\
& -2 x^{T}(t) C^{T} u(t)-u^{T}(t)\left(D+D^{T}-\gamma I_{n N}\right) u(t) .
\end{aligned}
$$

According to (A1), we can obtain

$$
\begin{aligned}
x^{T}(t) \widehat{P} F(x(t)) & =\sum_{i=1}^{N} x_{i}^{T}(t) \operatorname{Pf}\left(x_{i}(t)\right) \\
& \leqslant \sum_{i=1}^{N}\left[-\eta x_{i}^{T}(t) x_{i}(t)+x_{i}^{T}(t) P \Delta x_{i}(t)\right] \\
& =x^{T}(t)\left(-\eta I_{n N}+\widehat{P} \widehat{\Delta}\right) x(t) .
\end{aligned}
$$

It follows from (9) and (18) that

$$
\begin{aligned}
\dot{V}(t) & -2 y^{T}(t) u(t)+\gamma u^{T}(t) u(t) \\
\leqslant & x^{T}(t)\left(-2 \eta I_{n N}+2 \widehat{P} \widehat{\Delta}+C^{T} Q C\right) x(t) \\
& +2 a x^{T}(t) \widehat{P}(G \otimes \Gamma) y(t-\tau(t)) \\
& +2 x^{T}(t)\left(\widehat{P} B+C^{T} Q D-C^{T}\right) u(t) \\
& -(1-\sigma) y^{T}(t-\tau(t)) Q y(t-\tau(t)) \\
& -u^{T}(t)\left(D+D^{T}-\gamma I_{n N}-D^{T} Q D\right) u(t) \\
= & \xi^{T}(t)\left(\begin{array}{ccc}
a(G \otimes \Gamma)^{T} \widehat{P} & -(1-\sigma) Q & 0 \\
W_{1} & 0 & W_{3}
\end{array}\right) \xi(t) \\
\leqslant & 0,
\end{aligned}
$$

where $\xi(t)=\left(x^{T}(t), y^{T}(t-\tau(t)), u^{T}(t)\right)^{T}$.

By integrating (19) with respect to $t$ over the time period 0 to $t_{p}$, we get

$$
\begin{aligned}
& 2 \int_{0}^{t_{p}} y^{T}(s) u(s) d s \\
& \quad \geqslant V\left(t_{p}\right)-V(0)+\gamma \int_{0}^{t_{p}} u^{T}(s) u(s) d s .
\end{aligned}
$$

From the definition of $V(t)$, we have $V\left(t_{p}\right) \geqslant 0$ and $V(0) \geqslant 0$. Thus,

$$
2 \int_{0}^{t_{p}} y^{T}(s) u(s) d s \geqslant-\beta^{2}+\gamma \int_{0}^{t_{p}} u^{T}(s) u(s) d s
$$

for all $t_{p} \geqslant 0, \beta=\sqrt{V(0)}$. The proof is completed.

Theorem 5. Let (A1) hold, and let $\dot{\tau}(t) \leqslant \sigma<1$. The complex network (1) is input passive if there exist two matrices $Z \geqslant 0$ and $Q \geqslant 0$ and a scalar $\gamma>0$ such that

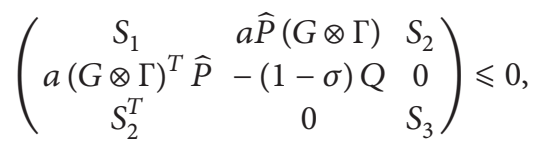

where

$$
\begin{gathered}
S_{1}=-2 \eta I_{n N}+2 \widehat{P} \widehat{\Delta}+C^{T}(Q+\tau Z) C, \\
S_{2}=\widehat{P} B-C^{T}+C^{T}(Q+\tau Z) D, \\
S_{3}=-\left[D+D^{T}-\gamma I_{n N}-D^{T}(Q+\tau Z) D\right] .
\end{gathered}
$$

Proof. Define the following Lyapunov functional for system (13):

$$
\begin{aligned}
V(t)= & x^{T}(t) \widehat{P} x(t)+\int_{-\tau(t)}^{0} \int_{t+\beta}^{t} y^{T}(\alpha) Z y(\alpha) d \alpha d \beta \\
& +\int_{t-\tau(t)}^{t} y^{T}(\alpha) Q y(\alpha) d \alpha .
\end{aligned}
$$

The derivative of $V(t)$ satisfies

$$
\begin{aligned}
\dot{V}(t)= & 2 x^{T}(t) \widehat{P} \dot{x}(t)+y^{T}(t) Q y(t) \\
& -\int_{t-\tau(t)}^{t} y^{T}(\alpha) Z y(\alpha) d \alpha+\tau(t) y^{T}(t) Z y(t) \\
& +\dot{\tau}(t) \int_{t-\tau(t)}^{t} y^{T}(\alpha) Z y(\alpha) d \alpha \\
& -(1-\dot{\tau}(t)) y^{T}(t-\tau(t)) Q y(t-\tau(t)) \\
\leqslant & 2 x^{T}(t) \widehat{P} \dot{x}(t)+y^{T}(t)(Q+\tau Z) y(t) \\
& -(1-\sigma) y^{T}(t-\tau(t)) Q y(t-\tau(t)) \\
= & 2 x^{T}(t) \widehat{P} F(x(t))+2 a x^{T}(t) \widehat{P}(G \otimes \Gamma) y(t-\tau(t)) \\
& +2 x^{T}(t) \widehat{P} B u(t)+y^{T}(t)(Q+\tau Z) y(t) \\
& -(1-\sigma) y^{T}(t-\tau(t)) Q y(t-\tau(t)) .
\end{aligned}
$$

Then, we can get

$$
\begin{aligned}
\dot{V}(t) & -2 y^{T}(t) u(t)+\gamma u^{T}(t) u(t) \\
\leqslant & 2 x^{T}(t) \widehat{P} F(x(t))+2 a x^{T}(t) \widehat{P}(G \otimes \Gamma) y(t-\tau(t)) \\
& +2 x^{T}(t)\left(\widehat{P} B-C^{T}\right) u(t)+y^{T}(t)(Q+\tau Z) y(t) \\
& -(1-\sigma) y^{T}(t-\tau(t)) Q y(t-\tau(t)) \\
& -u^{T}(t)\left(D+D^{T}-\gamma I_{n N}\right) u(t) .
\end{aligned}
$$

It follows from (18) and (22) that

$$
\begin{aligned}
\dot{V}(t) & -2 y^{T}(t) u(t)+\gamma u^{T}(t) u(t) \\
\leqslant & x^{T}(t)\left[-2 \eta I_{n N}+2 \widehat{P} \widehat{\Delta}+C^{T}(Q+\tau Z) C\right] x(t) \\
& +2 a x^{T}(t) \widehat{P}(G \otimes \Gamma) y(t-\tau(t)) \\
& +2 x^{T}(t)\left[\widehat{P} B-C^{T}+C^{T}(Q+\tau Z) D\right] u(t)
\end{aligned}
$$




$$
\begin{aligned}
& -u^{T}(t)\left[D+D^{T}-\gamma I_{n N}-D^{T}(Q+\tau Z) D\right] u(t) \\
& -(1-\sigma) y^{T}(t-\tau(t)) Q y(t-\tau(t)) \\
= & \xi^{T}(t)\left(\begin{array}{ccc}
S_{1} & a \widehat{P}(G \otimes \Gamma) & S_{2} \\
a(G \otimes \Gamma)^{T} \widehat{P} & -(1-\sigma) Q & 0 \\
S_{2}^{T} & 0 & S_{3}
\end{array}\right) \xi(t) \\
\leqslant & 0
\end{aligned}
$$

where $\xi(t)=\left(x^{T}(t), y^{T}(t-\tau(t)), u^{T}(t)\right)^{T}$.

By integrating (27) with respect to $t$ over the time period 0 to $t_{p}$, we can get

$$
\begin{aligned}
& 2 \int_{0}^{t_{p}} y^{T}(s) u(s) d s \\
& \quad \geqslant V\left(t_{p}\right)-V(0)+\gamma \int_{0}^{t_{p}} u^{T}(s) u(s) d s .
\end{aligned}
$$

From the definition of $V(t)$, we have $V\left(t_{p}\right) \geqslant 0$ and $V(0) \geqslant 0$. Thus,

$$
2 \int_{0}^{t_{p}} y^{T}(s) u(s) d s \geqslant-\beta^{2}+\gamma \int_{0}^{t_{p}} u^{T}(s) u(s) d s,
$$

for all $t_{p} \geqslant 0, \beta=\sqrt{V(0)}$. The proof is completed.

In the above, two sufficient conditions are given to ensure the input passivity of complex network (1). In the following, we shall discuss the output passivity of complex network (1).

Theorem 6. Let (A1) hold, and let $\dot{\tau}(t) \leqslant \sigma<1$. The complex network (1) is output passive if there exist matrix $Q \geqslant 0$ and scalar $\gamma>0$ such that

$$
\left(\begin{array}{ccc}
M_{1} & a \widehat{P}(G \otimes \Gamma) & M_{2} \\
a(G \otimes \Gamma)^{T} \widehat{P} & -(1-\sigma) Q & 0 \\
M_{2}^{T} & 0 & M_{3}
\end{array}\right) \leqslant 0
$$

where

$$
\begin{gathered}
M_{1}=-2 \eta I_{n N}+2 \widehat{P} \widehat{\Delta}+C^{T}\left(Q+\gamma I_{n N}\right) C, \\
M_{2}=\widehat{P} B+C^{T}\left(Q+\gamma I_{n N}\right) D-C^{T}, \\
M_{3}=-\left[D+D^{T}-D^{T}\left(Q+\gamma I_{n N}\right) D\right] .
\end{gathered}
$$

Proof. Construct the same Lyapunov functional as (15) for system (13). Then, we can get

$$
\begin{aligned}
\dot{V}(t) \leqslant & 2 x^{T}(t) \widehat{P} F(x(t))+2 a x^{T}(t) \widehat{P}(G \otimes \Gamma) y(t-\tau(t)) \\
& +2 x^{T}(t) \widehat{P} B u(t)+y^{T}(t) Q y(t) \\
& -(1-\sigma) y^{T}(t-\tau(t)) Q y(t-\tau(t)) .
\end{aligned}
$$

Therefore, we have

$$
\begin{aligned}
\dot{V}(t) & -2 y^{T}(t) u(t)+\gamma y^{T}(t) y(t) \\
\leqslant & 2 x^{T}(t) \widehat{P} F(x(t))+2 a x^{T}(t) \widehat{P}(G \otimes \Gamma) y(t-\tau(t)) \\
& +2 x^{T}(t)\left(\widehat{P} B-C^{T}\right) u(t)+y^{T}(t)\left(Q+\gamma I_{n N}\right) y(t) \\
& -(1-\sigma) y^{T}(t-\tau(t)) Q y(t-\tau(t)) \\
& -u^{T}(t)\left(D+D^{T}\right) u(t) .
\end{aligned}
$$

It follows from (18) and (30) that

$$
\begin{aligned}
\dot{V}(t) & -2 y^{T}(t) u(t)+\gamma y^{T}(t) y(t) \\
\leqslant & x^{T}(t)\left[-2 \eta I_{n N}+2 \widehat{P} \widehat{\Delta}+C^{T}\left(Q+\gamma I_{n N}\right) C\right] x(t) \\
& +2 a x^{T}(t) \widehat{P}(G \otimes \Gamma) y(t-\tau(t)) \\
& +2 x^{T}(t)\left[\widehat{P} B+C^{T}\left(Q+\gamma I_{n N}\right) D-C^{T}\right] u(t) \\
& -(1-\sigma) y^{T}(t-\tau(t)) Q y(t-\tau(t)) \\
& -u^{T}(t)\left[D+D^{T}-D^{T}\left(Q+\gamma I_{n N}\right) D\right] u(t) \\
= & \xi^{T}(t)\left(\begin{array}{ccc}
M_{1} & a \widehat{P}(G \otimes \Gamma) & M_{2} \\
a(G \otimes \Gamma)^{T} \widehat{P} & -(1-\sigma) Q & 0 \\
M_{2}^{T} & 0 & M_{3}
\end{array}\right) \xi(t) \\
\leqslant & 0,
\end{aligned}
$$

where $\xi(t)=\left(x^{T}(t), y^{T}(t-\tau(t)), u^{T}(t)\right)^{T}$.

By integrating (34) with respect to $t$ over the time period 0 to $t_{p}$, we get

$$
\begin{aligned}
& 2 \int_{0}^{t_{p}} y^{T}(s) u(s) d s \\
& \quad \geqslant V\left(t_{p}\right)-V(0)+\gamma \int_{0}^{t_{p}} y^{T}(s) y(s) d s .
\end{aligned}
$$

From the definition of $V(t)$, we have $V\left(t_{p}\right) \geqslant 0$ and $V(0) \geqslant 0$. Thus,

$$
2 \int_{0}^{t_{p}} y^{T}(s) u(s) d s \geqslant-\beta^{2}+\gamma \int_{0}^{t_{p}} y^{T}(s) y(s) d s
$$

for all $t_{p} \geqslant 0, \beta=\sqrt{V(0)}$. The proof is completed.

Theorem 7. Let (A1) hold, and let $\dot{\tau}(t) \leqslant \sigma<1$. The complex network (1) is output passive if there exist two matrices $Z \geqslant 0$ and $Q \geqslant 0$ and a scalar $\gamma>0$ such that

$$
\left(\begin{array}{ccc}
H_{1} & a \widehat{P}(G \otimes \Gamma) & H_{2} \\
a(G \otimes \Gamma)^{T} \widehat{P} & -(1-\sigma) Q & 0 \\
H_{2}^{T} & 0 & H_{3}
\end{array}\right) \leqslant 0,
$$


where

$$
\begin{gathered}
H_{1}=-2 \eta I_{n N}+2 \widehat{P} \widehat{\Delta}+C^{T}\left(Q+\tau Z+\gamma I_{n N}\right) C, \\
H_{2}=\widehat{P} B-C^{T}+C^{T}\left(Q+\tau Z+\gamma I_{n N}\right) D, \\
H_{3}=-\left[D+D^{T}-D^{T}\left(Q+\tau Z+\gamma I_{n N}\right) D\right] .
\end{gathered}
$$

Proof. Construct the same Lyapunov functional as (24) for system (13). Then, we can obtain

$$
\begin{aligned}
\dot{V}(t) \leqslant & 2 x^{T}(t) \hat{P} F(x(t))+2 a x^{T}(t) \widehat{P}(G \otimes \Gamma) y(t-\tau(t)) \\
& +2 x^{T}(t) \widehat{P} B u(t)+y^{T}(t)(Q+\tau Z) y(t) \\
& -(1-\sigma) y^{T}(t-\tau(t)) Q y(t-\tau(t)) .
\end{aligned}
$$

Therefore, we have

$$
\begin{aligned}
\dot{V}(t) & -2 y^{T}(t) u(t)+\gamma y^{T}(t) y(t) \\
\leqslant & 2 x^{T}(t) \widehat{P} F(x(t))+2 a x^{T}(t) \widehat{P}(G \otimes \Gamma) y(t-\tau(t)) \\
& +2 x^{T}(t)\left(\widehat{P} B-C^{T}\right) u(t)+y^{T}(t)\left(Q+\tau Z+\gamma I_{n N}\right) y(t) \\
& -(1-\sigma) y^{T}(t-\tau(t)) Q y(t-\tau(t)) \\
& -u^{T}(t)\left(D+D^{T}\right) u(t) .
\end{aligned}
$$

It follows from (18) and (37) that

$$
\begin{aligned}
\dot{V}(t) & -2 y^{T}(t) u(t)+\gamma y^{T}(t) y(t) \\
\leqslant & x^{T}(t)\left[-2 \eta I_{n N}+2 \widehat{P} \widehat{\Delta}+C^{T}\left(Q+\tau Z+\gamma I_{n N}\right) C\right] x(t) \\
& +2 a x^{T}(t) \widehat{P}(G \otimes \Gamma) y(t-\tau(t)) \\
& +2 x^{T}(t)\left[\widehat{P} B-C^{T}+C^{T}\left(Q+\tau Z+\gamma I_{n N}\right) D\right] u(t) \\
& -(1-\sigma) y^{T}(t-\tau(t)) Q y(t-\tau(t)) \\
& -u^{T}(t)\left[D+D^{T}-D^{T}\left(Q+\tau Z+\gamma I_{n N}\right) D\right] u(t) \\
= & \xi^{T}(t)\left(\begin{array}{ccc}
H_{1} & a \widehat{P}(G \otimes \Gamma) & H_{2} \\
a(G \Gamma)^{T} \widehat{P} & -(1-\sigma) Q & 0 \\
H_{2}^{T} & 0 & H_{3}
\end{array}\right) \xi(t) \\
\leqslant & 0,
\end{aligned}
$$

where $\xi(t)=\left(x^{T}(t), y^{T}(t-\tau(t)), u^{T}(t)\right)^{T}$.

By integrating (41) with respect to $t$ over the time period 0 to $t_{p}$, we get

$$
\begin{aligned}
& 2 \int_{0}^{t_{p}} y^{T}(s) u(s) d s \\
& \quad \geqslant V\left(t_{p}\right)-V(0)+\gamma \int_{0}^{t_{p}} y^{T}(s) y(s) d s .
\end{aligned}
$$

From the definition of $V(t)$, we have $V\left(t_{p}\right) \geqslant 0$ and $V(0) \geqslant 0$. Thus,

$$
2 \int_{0}^{t_{p}} y^{T}(s) u(s) d s \geqslant-\beta^{2}+\gamma \int_{0}^{t_{p}} y^{T}(s) y(s) d s,
$$

for all $t_{p} \geqslant 0, \beta=\sqrt{V(0)}$. The proof is completed.

Remark 8. In recent years, some researchers have studied the input passivity and output passivity of the complex networks with state coupling, and many interesting results have been derived. To the best of our knowledge, this is the first paper to investigate the input passivity and output passivity of complex delayed dynamical networks with output coupling. By constructing new Lyapunov functionals, some sufficient conditions ensuring the input passivity and output passivity are established in this paper.

\section{Examples}

In this section, two illustrative examples are provided to verify the effectiveness of the proposed theoretical results.

Example 1. Consider a three-order dynamical system as the dynamical node of the complex network (1) which is described by

$$
\left(\begin{array}{c}
\dot{x}_{1} \\
\dot{x}_{2} \\
\dot{x}_{3}
\end{array}\right)=\left(\begin{array}{c}
-10 x_{1}+2 x_{2} \\
2 x_{1}-10 x_{2}-x_{1} x_{3} \\
x_{1} x_{2}-6 x_{3}
\end{array}\right) \text {. }
$$

Clearly, we can take $\eta=6, P=\operatorname{diag}(1,1,1)$, and $\Delta=$ $\operatorname{diag}(0,0,0)$. Take

$$
\begin{aligned}
\Gamma=\left(\begin{array}{lll}
0.4 & 0.2 & 0.2 \\
0.3 & 0.2 & 0.3 \\
0.3 & 0.1 & 0.2
\end{array}\right), & C_{i}=\left(\begin{array}{ccc}
0.3 & 0.2 & 0.1 \\
0.4 & 0.1 & 0.5 \\
0.4 & 0.3 & 0.2
\end{array}\right), \\
B_{i}=\left(\begin{array}{ccc}
0.3 & 0.1 & 0.1 \\
0.4 & 0.1 & 0.3 \\
0.7 & 0 & 0.2
\end{array}\right), & D_{i}=\left(\begin{array}{ccc}
2.5 & 0 & 0 \\
0 & 3.6 & 0 \\
0 & 0 & 2.6
\end{array}\right),
\end{aligned}
$$

$a=0.2, \beta_{\omega}=1$, and $i=1,2, \ldots, 10$. The matrix $L$ is chosen as follows:

$$
\left(\begin{array}{cccccccccc}
-3 & 1 & 1 & 0 & 0 & 0 & 0 & 0 & 0 & 1 \\
1 & -4 & 1 & 0 & 1 & 0 & 1 & 0 & 0 & 0 \\
1 & 1 & -5 & 1 & 0 & 1 & 0 & 0 & 0 & 1 \\
0 & 0 & 1 & -3 & 1 & 0 & 0 & 0 & 1 & 0 \\
0 & 1 & 0 & 1 & -3 & 1 & 0 & 0 & 0 & 0 \\
0 & 0 & 1 & 0 & 1 & -3 & 1 & 0 & 0 & 0 \\
0 & 1 & 0 & 0 & 0 & 1 & -4 & 1 & 0 & 1 \\
0 & 0 & 0 & 0 & 0 & 0 & 1 & -2 & 1 & 0 \\
0 & 0 & 0 & 1 & 0 & 0 & 0 & 1 & -2 & 0 \\
1 & 0 & 1 & 0 & 0 & 0 & 1 & 0 & 0 & -3
\end{array}\right)
$$


Obviously, network (1) is connected, and matrix $G$ is

$$
\left(\begin{array}{cccccccccc}
-1 & \frac{1}{3} & \frac{1}{3} & 0 & 0 & 0 & 0 & 0 & 0 & \frac{1}{3} \\
\frac{1}{4} & -1 & \frac{1}{4} & 0 & \frac{1}{4} & 0 & \frac{1}{4} & 0 & 0 & 0 \\
\frac{1}{5} & \frac{1}{5} & -1 & \frac{1}{5} & 0 & \frac{1}{5} & 0 & 0 & 0 & \frac{1}{5} \\
0 & 0 & \frac{1}{3} & -1 & \frac{1}{3} & 0 & 0 & 0 & \frac{1}{3} & 0 \\
0 & \frac{1}{3} & 0 & \frac{1}{3} & -1 & \frac{1}{3} & 0 & 0 & 0 & 0 \\
0 & 0 & \frac{1}{3} & 0 & \frac{1}{3} & -1 & \frac{1}{3} & 0 & 0 & 0 \\
0 & \frac{1}{4} & 0 & 0 & 0 & \frac{1}{4} & -1 & \frac{1}{4} & 0 & \frac{1}{4} \\
0 & 0 & 0 & 0 & 0 & 0 & \frac{1}{2} & -1 & \frac{1}{2} & 0 \\
0 & 0 & 0 & \frac{1}{2} & 0 & 0 & 0 & \frac{1}{2} & -1 & 0 \\
\frac{1}{3} & 0 & \frac{1}{3} & 0 & 0 & 0 & \frac{1}{3} & 0 & 0 & -1
\end{array}\right)
$$
(1).

Next, we analyze the input passivity of complex network

Setting $\tau(t)=0.5-0.5 e^{-t}$, then $0 \leqslant \tau(t) \leqslant \tau=0.5$ and $\dot{\tau}(t)=0.5 e^{-t} \leqslant 0.5$, for $t \geqslant 0$.

We can find the following matrix $Q$ satisfying (9) with $\gamma=$ 0.2. Consider the following:

$Q=\operatorname{diag}(0.5341,0.4135,0.3429,0.5341,0.4135,0.3429$,

$0.5341,0.4135,0.3429,0.5341,0.4135,0.3429$,

$0.5341,0.4135,0.3429,0.5341,0.4135,0.3429$,

0.5341, 0.4135, 0.3429, 0.5341, 0.4135, 0.3429,

$0.5341,0.4135,0.3429,0.5341,0.4135,0.3429)$.

Hence, it follows from Theorem 4 that complex network (1) with above given parameters is input passive.

Example 2. Consider a three-order dynamical system as the dynamical node of the complex network (1) which is described by

$$
\left(\begin{array}{c}
\dot{x}_{1} \\
\dot{x}_{2} \\
\dot{x}_{3}
\end{array}\right)=\left(\begin{array}{c}
-9 x_{1}+2 x_{2} \\
x_{1}-9 x_{2}-x_{1} x_{3} \\
x_{1} x_{2}-7 x_{3}
\end{array}\right)
$$

Clearly, we can take $\eta=7, P=\operatorname{diag}(1,1,1)$, and $\Delta=$ $\operatorname{diag}(0,0,0)$. Take

$$
\Gamma=\left(\begin{array}{lll}
0.1 & 0.4 & 0.6 \\
0.3 & 0.4 & 0.1 \\
0.3 & 0.5 & 0.2
\end{array}\right), \quad C_{i}=\left(\begin{array}{lll}
0.6 & 0.3 & 0.4 \\
0.2 & 0.1 & 0.3 \\
0.1 & 0.3 & 0.7
\end{array}\right)
$$

$$
B_{i}=\left(\begin{array}{ccc}
0.1 & 0.5 & 0.3 \\
0.5 & 0.3 & 0.1 \\
0.2 & 0.7 & 0.2
\end{array}\right), \quad D_{i}=\left(\begin{array}{ccc}
2.9 & 0 & 0 \\
0 & 3.3 & 0 \\
0 & 0 & 2.8
\end{array}\right)
$$

$a=0.3, \beta_{\omega}=1$, and $i=1,2, \ldots, 10$. The matrix $L$ is chosen as follows:

$$
\left(\begin{array}{cccccccccc}
-2 & 0 & 0 & 0 & 0 & 0 & 0 & 0 & 1 & 1 \\
0 & -1 & 1 & 0 & 0 & 0 & 0 & 0 & 0 & 0 \\
0 & 1 & -2 & 0 & 0 & 0 & 0 & 0 & 0 & 1 \\
0 & 0 & 0 & -1 & 0 & 0 & 0 & 0 & 0 & 1 \\
0 & 0 & 0 & 0 & -1 & 0 & 0 & 0 & 0 & 1 \\
0 & 0 & 0 & 0 & 0 & -1 & 0 & 0 & 1 & 0 \\
0 & 0 & 0 & 0 & 0 & 0 & -1 & 1 & 0 & 0 \\
0 & 0 & 0 & 0 & 0 & 0 & 1 & -2 & 1 & 0 \\
1 & 0 & 0 & 0 & 0 & 1 & 0 & 1 & -4 & 1 \\
1 & 0 & 1 & 1 & 1 & 0 & 0 & 0 & 1 & -5
\end{array}\right) .
$$

Obviously, network (1) is connected, and matrix $G$ is

$$
\left(\begin{array}{cccccccccc}
-1 & 0 & 0 & 0 & 0 & 0 & 0 & 0 & \frac{1}{2} & \frac{1}{2} \\
0 & -1 & 1 & 0 & 0 & 0 & 0 & 0 & 0 & 0 \\
0 & \frac{1}{2} & -1 & 0 & 0 & 0 & 0 & 0 & 0 & \frac{1}{2} \\
0 & 0 & 0 & -1 & 0 & 0 & 0 & 0 & 0 & 1 \\
0 & 0 & 0 & 0 & -1 & 0 & 0 & 0 & 0 & 1 \\
0 & 0 & 0 & 0 & 0 & -1 & 0 & 0 & 1 & 0 \\
0 & 0 & 0 & 0 & 0 & 0 & -1 & 1 & 0 & 0 \\
0 & 0 & 0 & 0 & 0 & 0 & \frac{1}{2} & -1 & \frac{1}{2} & 0 \\
\frac{1}{4} & 0 & 0 & 0 & 0 & \frac{1}{4} & 0 & \frac{1}{4} & -1 & \frac{1}{4} \\
\frac{1}{5} & 0 & \frac{1}{5} & \frac{1}{5} & \frac{1}{5} & 0 & 0 & 0 & \frac{1}{5} & -1
\end{array}\right) .
$$

In the following, we analyze the output passivity of complex network (1).

Setting $\tau(t)=0.5-0.5 e^{-t}$, then $0 \leqslant \tau(t) \leqslant \tau=0.5$ and $\dot{\tau}(t)=0.5 e^{-t} \leqslant 0.5$, for $t \geqslant 0$.

We can find the following matrix $Q$ satisfying (30) with $\gamma=0.1$. Consider the following:

$Q=\operatorname{diag}(0.3548,0.3893,0.1592,0.3548,0.3893,0.1592$, $0.3548,0.3893,0.1592,0.3548,0.3893,0.1592$, $0.3548,0.3893,0.1592,0.3548,0.3893,0.1592$, $0.3548,0.3893,0.1592,0.3548,0.3893,0.1592$, $0.3548,0.3893,0.1592,0.3548,0.3893,0.1592)$.

By Theorem 6, we know that complex network (1) with above given parameters is output passive.

\section{Conclusion}

A new complex delayed dynamical network model with output coupling has been introduced. We have considered 
the input passivity and output passivity of the proposed network model. Some input passivity and output passivity criteria have been established by constructing new Lyapunov functionals. Moreover, two illustrative examples have been provided to verify the correctness and effectiveness of the obtained results. In future work, we shall study the input passivity and output passivity of impulsive complex delayed dynamical networks with output coupling.

\section{Conflict of Interests}

The authors declare that there is no conflict of interests regarding the publication of this paper.

\section{Acknowledgments}

The authors would like to thank the Associate Editor and anonymous reviewers for their valuable comments and suggestions. This work was supported in part by the Fundamental Research Funds for the Central University (2013-YB-017).

\section{References}

[1] J. Wang and $\mathrm{H}$. Wu, "Synchronization criteria for impulsive complex dynamical networks with time-varying delay," Nonlinear Dynamics, vol. 70, no. 1, pp. 13-24, 2012.

[2] J.-L. Wang and H.-N. Wu, "Stability analysis of impulsive parabolic complex networks," Chaos, Solitons and Fractals, vol. 44, no. 11, pp. 1020-1034, 2011.

[3] J.-L. Wang, Z.-C. Yang, T.-W. Huang, and M.-Q. Xiao, "Local and global exponential synchronization of complex delayed dynamical networks with general topology," Discrete and Continuous Dynamical Systems B, vol. 16, no. 1, pp. 393-408, 2011.

[4] J. Wang, Z. Yang, T. Huang, and M. Xiao, "Synchronization criteria in complex dynamical networks with nonsymmetric coupling and multiple time-varying delays," Applicable Analysis, vol. 91, no. 5, pp. 923-935, 2012.

[5] T. Huang, G. Chen, and J. Kurths, "Synchronization of chaotic systems with time-varying coupling delays," Discrete and Continuous Dynamical Systems B: A Journal Bridging Mathematics and Sciences, vol. 16, no. 4, pp. 1071-1082, 2011.

[6] W. Yu, G. Chen, and M. Cao, "Consensus in directed networks of agents with nonlinear dynamics," IEEE Transactions on Automatic Control, vol. 56, no. 6, pp. 1436-1441, 2011.

[7] W. W. Yu, J. D. Cao, and J. H. Lü, "Global synchronization of linearly hybrid coupled networks with time-varying delay," SIAM Journal on Applied Dynamical Systems, vol. 7, no. 1, pp. 108-133, 2008.

[8] X. Yang, J. Cao, and J. Lu, "Stochastic synchronization of complex networks with nonidentical nodes via hybrid adaptive and impulsive control," IEEE Transactions on Circuits and Systems. I. Regular Papers, vol. 59, no. 2, pp. 371-384, 2012.

[9] G. Wang, J. Cao, and J. Lu, "Outer synchronization between two nonidentical networks with circumstance noise," Physica A, vol. 389, no. 7, pp. 1480-1488, 2010.

[10] L. Y. Xiang and J. J. H. Zhu, "On pinning synchronization of general coupled networks," Nonlinear Dynamics, vol. 64, no. 4, pp. 339-348, 2011.

[11] G. P. Jiang, W. K. S. Tang, and G. R. Chen, "A state-observerbased approach for synchronization in complex dynamical networks," IEEE Transactions on Circuits and Systems I: Regular Papers, vol. 53, no. 12, pp. 2739-2745, 2006.

[12] Y. Gao and L. Wang, "Consensus of multiple dynamic agents with sampled information," IET Control Theory \& Applications, vol. 4, no. 6, pp. 945-956, 2010.

[13] J. L. Wang and H. N. Wu, "Local and global exponential output synchronization of complex delayed dynamical networks," Nonlinear Dynamics, vol. 67, no. 1, pp. 497-504, 2012.

[14] J. L. Wang and H. N. Wu, "Adaptive output synchronization of complex delayed dynamical networks with output coupling," Neurocomputing, vol. 142, pp. 174-181, 2014.

[15] J. L. Wang and H. N. Wu, "Passivity of delayed reactiondiffusion networks with application to a food web model," Applied Mathematics and Computation, vol. 219, no. 24, pp. 11311-11326, 2013.

[16] R. Lozano, B. Brogliato, O. Egeland, and B. Maschke, Dissipative Systems Analysis and Control: Theory and Applications, Communications and Control Engineering Series, Springer, London, UK, 2000.

[17] H. Gao, T. Chen, and T. Chai, "Passivity and passification for networked control systems," SIAM Journal on Control and Optimization, vol. 46, no. 4, pp. 1299-1322, 2007.

[18] J. L. Chen and L. Lee, "Passivity approach to feedback connection stability for discrete-time descriptor systems," in Proceeding of the 40th IEEE Conference on Decision and Control (CDC '01), vol. 3, pp. 2865-2866, Orlando, Fla, USA, December 2001.

[19] X. W. Liu, "Passivity analysis of uncertain fuzzy delayed systems," Chaos, Solitons and Fractals, vol. 34, no. 3, pp. 833-838, 2007.

[20] J. Liang, Z. Wang, and X. Liu, "Robust passivity and passification of stochastic fuzzy time-delay systems," Information Sciences, vol. 180, no. 9, pp. 1725-1737, 2010.

[21] W. J. Chang, C. C. Ku, and P. H. Huang, "Fuzzy controller design for passive continuous-time affine T-S fuzzy models with relaxed stability conditions," ISA Transactions, vol. 48, no. 3, pp. 295-303, 2009.

[22] C. Li, H. Zhang, and X. Liao, "Passivity and passification of fuzzy systems with time delays," Computers \& Mathematics with Applications, vol. 52, no. 6-7, pp. 1067-1078, 2006.

[23] X. Y. Lou and B. T. Cui, "Passivity analysis of integro-differential neural networks with time-varying delays," Neurocomputing, vol. 70, no. 4-6, pp. 1071-1078, 2007.

[24] J. H. Park, "Further results on passivity analysis of delayed cellular neural networks," Chaos, Solitons and Fractals, vol. 34, no. 5, pp. 1546-1551, 2007.

[25] B. Chen, H. Li, C. Lin, and Q. Zhou, "Passivity analysis for uncertain neural networks with discrete and distributed timevarying delays," Physics Letters A, vol. 373, no. 14, pp. 1242-1248, 2009.

[26] Q. Song, J. Liang, and Z. Wang, "Passivity analysis of discretetime stochastic neural networks with time-varying delays," Neurocomputing, vol. 72, no. 7-9, pp. 1782-1788, 2009.

[27] Q. Song and Z. Wang, "New results on passivity analysis of uncertain neural networks with time-varying delays," International Journal of Computer Mathematics, vol. 87, no. 1-3, pp. 668-678, 2010.

[28] C. G. Li and X. F. Liao, "Passivity analysis of neural networks with time delay," IEEE Transactions on Circuits and Systems II: Express Briefs, vol. 52, no. 8, pp. 471-475, 2005.

[29] J. Yao, H. O. Wang, Z. H. Guan, and W. S. Xu, "Passive stability and synchronization of complex spatio-temporal switching 
networks with time delays," Automatica, vol. 45, no. 7, pp. 17211728, 2009.

[30] J. Yao, Z. H. Guan, and D. J. Hill, "Passivity-based control and synchronization of general complex dynamical networks," Automatica, vol. 45, no. 9, pp. 2107-2113, 2009.

[31] J. L. Wang, H. N. Wu, and L. Guo, "Passivity and stability analysis of reaction-diffusion neural networks with dirichlet boundary conditions," IEEE Transactions on Neural Networks, vol. 22, no. 12, pp. 2105-2116, 2011.

[32] J.-L. Wang, Z.-C. Yang, and H.-N. Wu, "Passivity analysis of complex dynamical networks with multiple time-varying delays," Journal of Engineering Mathematics, vol. 74, pp. 175-188, 2012.

[33] J.-L. Wang and H.-N. Wu, "Robust stability and robust passivity of parabolic complex networks with parametric uncertainties and time-varying delays," Neurocomputing, vol. 87, pp. 26-32, 2012.

[34] A. J. Laub, Matrix Analysis for Scientists and Engineers, Society for Industrial and Applied Mathematics, Philadelphia, PA, USA, 2005.

[35] W. L. Guo, F. Austin, and S. H. Chen, "Global synchronization of nonlinearly coupled complex networks with non-delayed and delayed coupling," Communications in Nonlinear Science and Numerical Simulation, vol. 15, no. 6, pp. 1631-1639, 2010. 


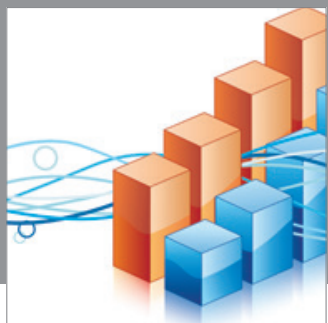

Advances in

Operations Research

mansans

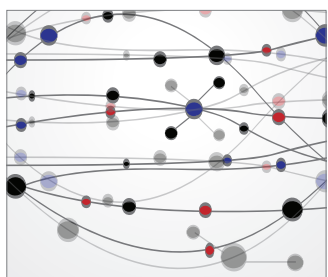

The Scientific World Journal
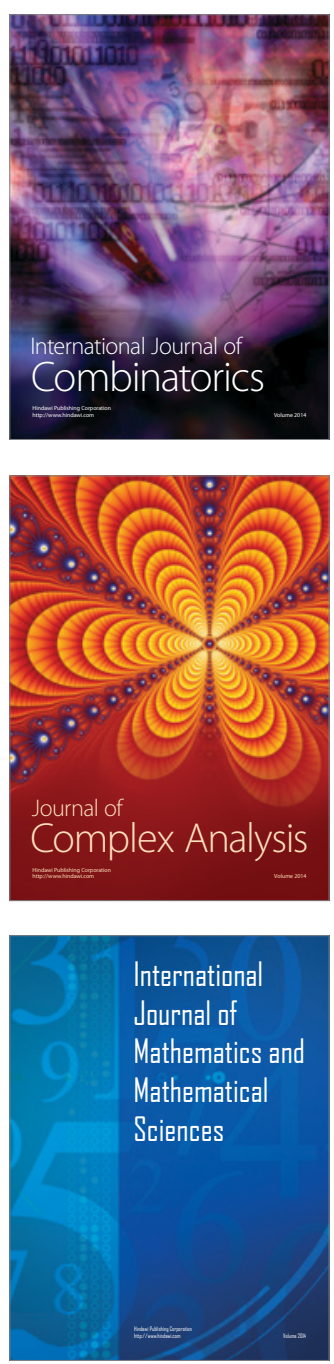
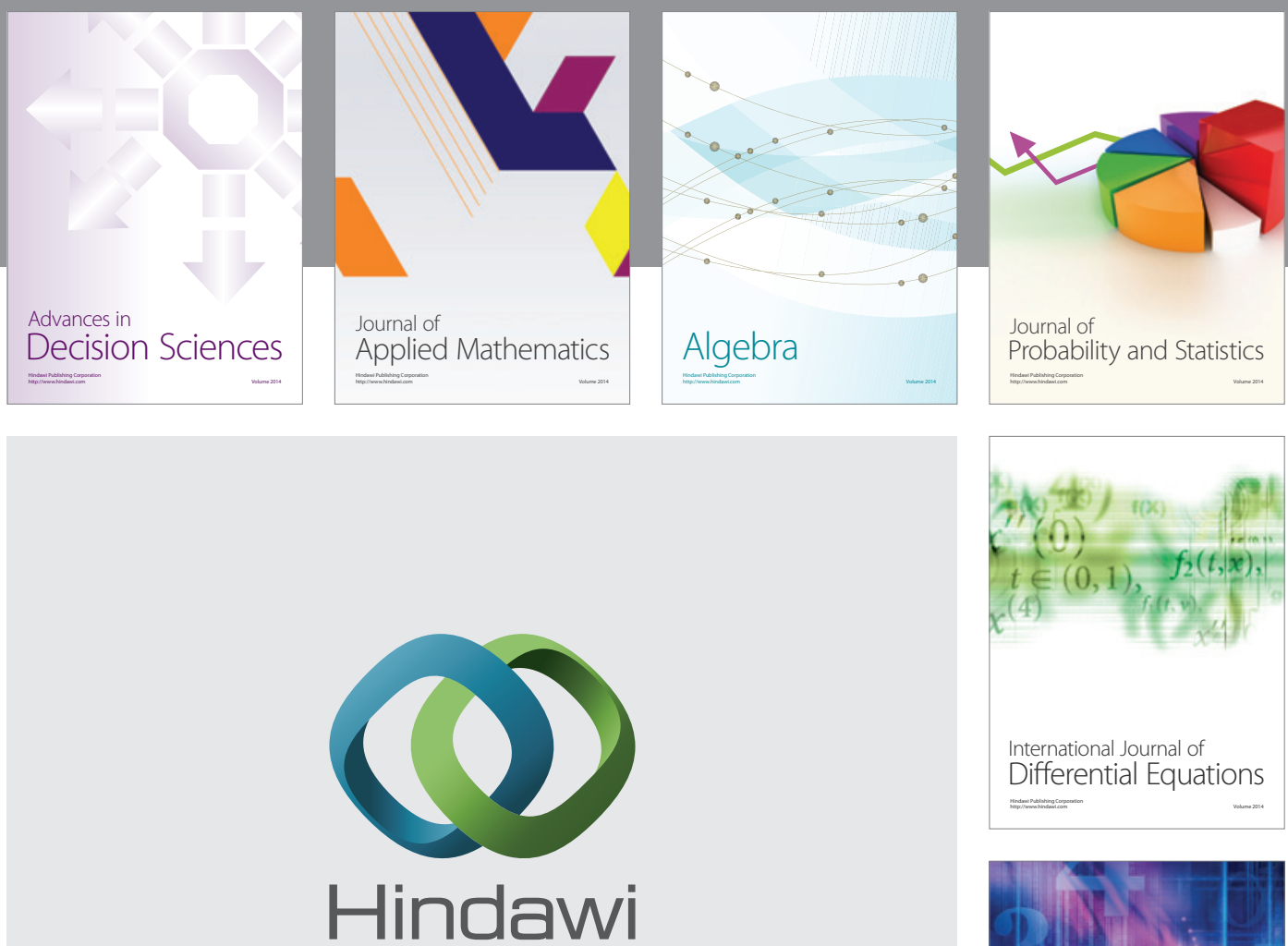

Submit your manuscripts at http://www.hindawi.com
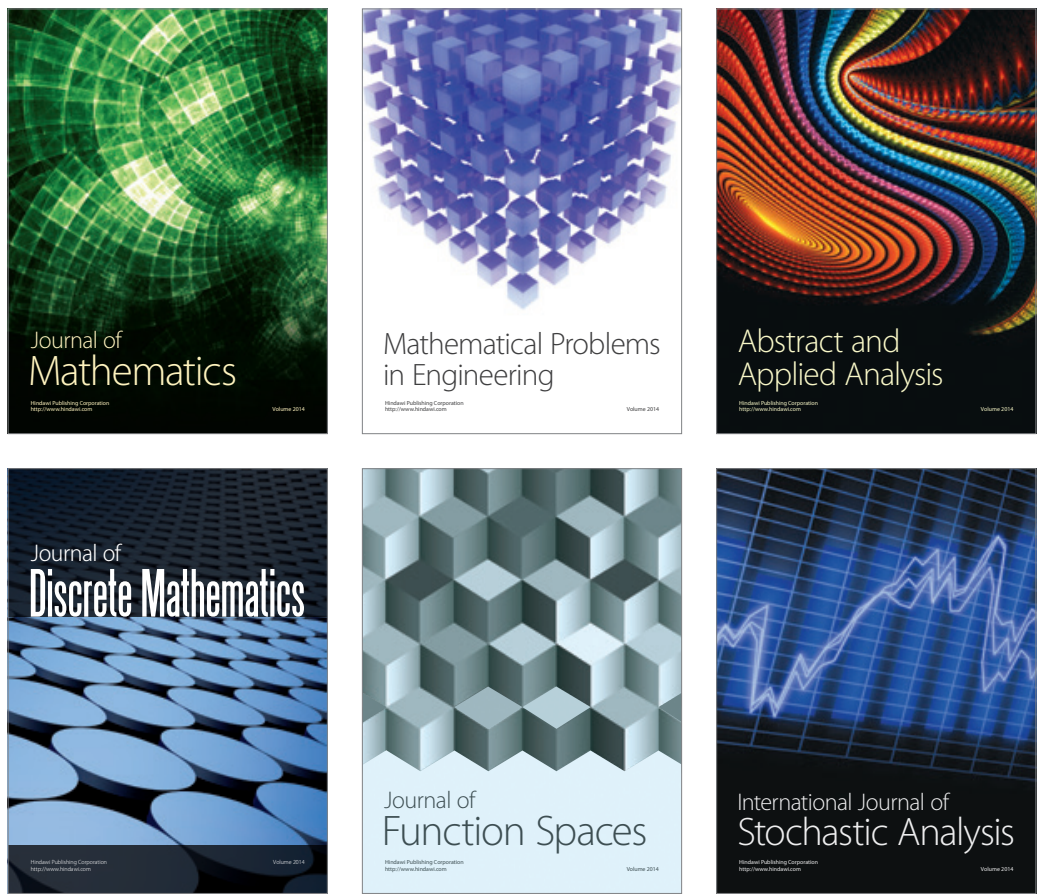

Journal of

Function Spaces

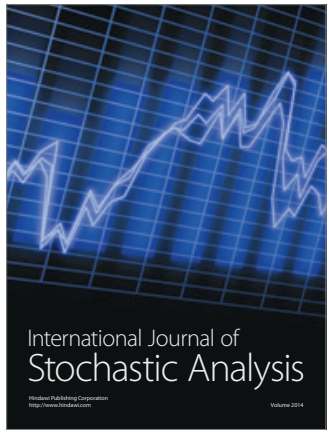

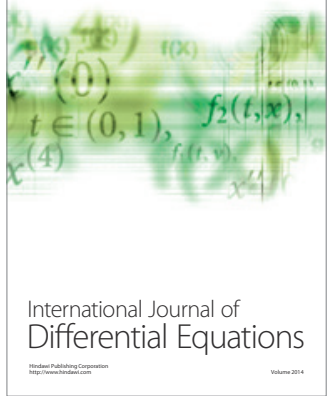
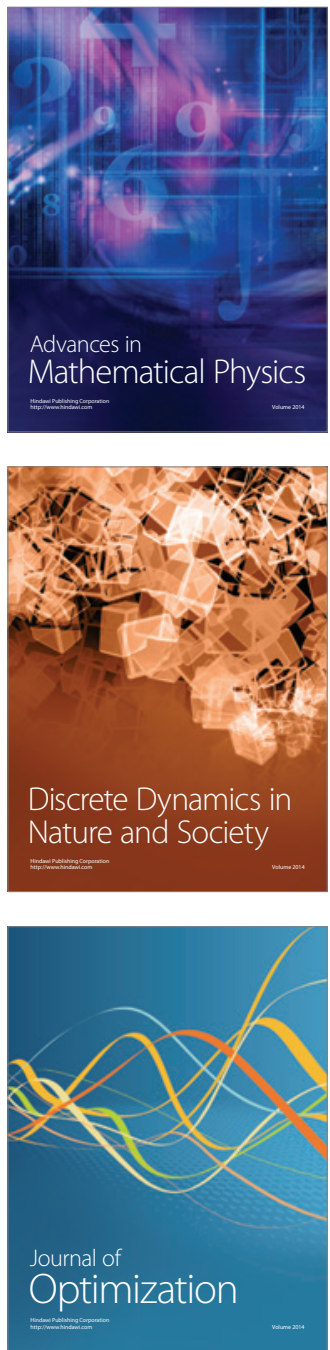